\title{
Coccygectomy as a Surgical Option in the Treatment of Chronic Traumatic Coccygodynia
}

\author{
Omer Ersen ${ }^{1}$, Safak Ekinci ${ }^{2}$, Kenan Koca $^{3}$, Faruk Akyildiz ${ }^{4}$, Serkan Bilgic ${ }^{5}$ \\ ${ }^{1}$ Department of Orthopaedic Surgery, Erzurum Military Hospital, Erzurum, Turkey \\ ${ }^{2}$ Department of Orthopaedic Surgery, Agri Military Hospital, Agri, Turkey \\ ${ }^{3}$ Department of Orthopaedic Surgery, Gulhane Military Hospital, Ankara, Turkey \\ ${ }^{4}$ Department of Orthopaedic Surgery, Malatya Military Hospital, Malatya, Turkey \\ ${ }^{5}$ Department of Orthopaedic Surgery, Haydarpasa Gulhane Military Hospital, Istanbul, Turkey
}

Dear editor,

We recently read the interesting article entitled "Coccygectomy as a surgical option in the treatment of chronic traumatic coccygodynia: a single-center experience and literature review" by Antoniadis et al. [1] which touches on an underrated topic in neurosurgical practice.

Periosteal preservation and closure during the coccygectomy procedure have been found to be related to a low risk of infection [2]. Although Antoniadis et al. did not mention this in their case series, the information should be presented in a literature review.

Additionally, some other treatment options, such as caudal epidural pulsed radiofrequency treatment, have been found to be efficient after unsuccessful non-surgical and surgical treatment modalities in the treatment of coccygodynia and should also be mentioned in the article. Atim et al. [3] achieved excellent and good results in 81\% of cases treated with caudal epidural pulsed radiofrequency.
We expect that these small additions will increase the value of Antoniadis' article.

\section{Conflict of Interest}

No potential conflict of interest relevant to this article was reported.

\section{References}

1. Antoniadis A, Ulrich NH, Senyurt H. Coccygectomy as a surgical option in the treatment of chronic traumatic coccygodynia: a single-center experience and literature review. Asian Spine J 2014;8:705-10.

2. Bilgic S, Kurklu M, Yurttas Y, Ozkan H, Oguz E, Sehirlioglu A. Coccygectomy with or without periosteal resection. Int Orthop 2010;34:537-41.

3. Atim A, Ergin A, Bilgic S, Deniz S, Kurt E. Pulsed radiofrequency in the treatment of coccygodynia. Agri 2011;23:1-6. 